See discussions, stats, and author profiles for this publication at: https://www.researchgate.net/publication/319348072

\title{
Multimode time-dependent gyrotron equations for different time scales
}

Article in Physics of Plasmas · September 2017

DOl: $10.1063 / 1.5000388$

CITATION

1

2 authors:

O. Dumbrajs

University of Latvia

299 PUBLICATIONS 3,465 CITATIONS

SEE PROFILE
READS

71

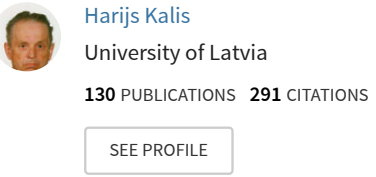

Some of the authors of this publication are also working on these related projects:

Improvement and control of pelletized biomass combustion process dynamics in electric field View project

Co-firing of biomass with electrodynamic process control for clean and effective energy production View project 
Multimode time-dependent gyrotron equations for different time scales

O. Dumbrajs, and H. Kalis

Citation: Physics of Plasmas 24, 093111 (2017); doi: 10.1063/1.5000388

View online: http://dx.doi.org/10.1063/1.5000388

View Table of Contents: http://aip.scitation.org/toc/php/24/9

Published by the American Institute of Physics

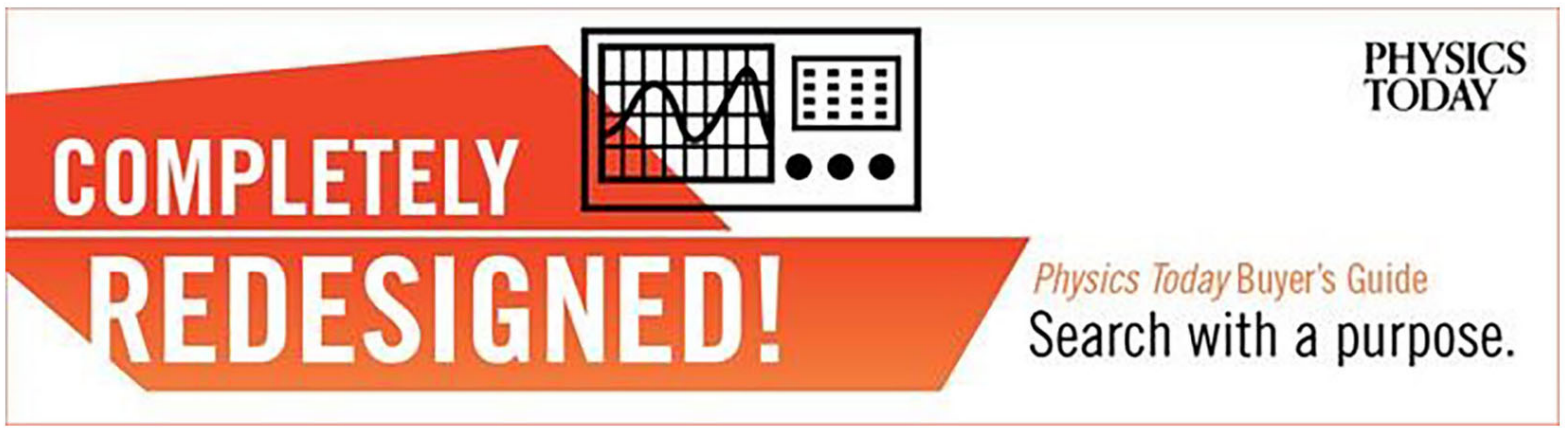




\title{
Multimode time-dependent gyrotron equations for different time scales
}

\author{
O. Dumbrajs ${ }^{1}$ and H. Kalis ${ }^{2}$ \\ ${ }^{1}$ Institute of Solid State Physics, University of Latvia, Kengaraga Street 8, LV-1063 Riga, Latvia \\ ${ }^{2}$ Institute of Mathematics and Computer Science of University of Latvia, Raing bulv. 29, LV-1459 Riga, Latvia
}

(Received 27 April 2017; accepted 15 August 2017; published online 29 August 2017)

\begin{abstract}
Development of gyrotrons requires careful understanding of different regimes of gyrotron oscillations. For a long time, the gyrotron theory was developed assuming that the transit time of electrons through the interaction space is much shorter than the cavity fill time. Correspondingly, it was assumed that during this transit time, the amplitude of microwave oscillations remains constant. However, there are situations when this assumption is not fulfilled, or is marginally fulfilled. In such cases, a different mathematical formalism has to be used. The present paper generalizes the new formalism to the multi mode case. The particular example considered indicates that in some cases the results obtained by means of the old and the new formalism differ significantly. Published by AIP Publishing. [http://dx.doi.org/10.1063/1.5000388]
\end{abstract}

\section{INTRODUCTION}

In Ref. 1, we applied the new formalism to analyze different regimes of oscillations in the plane of normalized parameters characterizing the external magnetic field and the beam current. In this plane, complicated alternating sequences of regions of stationary, periodic, auto modulation, and chaotic oscillations exist. In the past, these regions were investigated on the supposition that the transit time of electrons through the interaction space is much shorter than the cavity decay time (Ref. 2). Our studies revealed that in most of the cases the results obtained by the old and the new formalism are different.

In Ref. 3, the self-consistent non-stationary theory of the gyrotron was generalized using the new formalism. The main attention was paid to modification of the boundary between the regions of oscillations with constant amplitude and auto modulation. It was shown that the theory free from the assumption about the frozen wave amplitude during the electron transit time predicts some widening of the region of auto modulation.

In the present paper, we extend the new formalism to the case of multimode interactions.

The paper is organized as follows. In Sec. II, we recall the formalism used earlier to study self-consistently nonstationary multimode oscillations in gyrotrons. Then, in Sec. III we describe the new formalism which has to be used, when the inequality $t_{\text {transit }} \ll t_{\text {decay }}$ is violated, or is marginally fulfilled. In Sec. IV, we present an example of simulations on the basis of the new formalism and compare the new results with those obtained by means of the old formalism. Section V summarizes the study.

\section{OLD FORMALISM}

If the electron transit time through the resonator is much shorter than the cavity decay time $t_{\text {transit }} \ll t_{\text {decay }}$ ( $t_{\text {transit }}=L / v_{z}$, $t_{\text {decay }} \sim Q / \omega, Q$ is the quality factor, $\omega$ is the oscillation frequency, $L$ is the interaction length, and $v_{z}$ is the longitudinal velocity), one can use the formalism developed in Ref. 4 which is available only in Russian. Therefore, it is expedient to repeat it

$$
\begin{gathered}
\frac{\partial^{2} f_{s}}{\partial \varsigma^{2}}-i n_{s} \frac{\partial f_{s}}{\partial \tau}+n_{s} \delta_{s} f_{s}=I_{s} \frac{1}{4 \pi^{2}} \int_{0}^{2 \pi} \int_{0}^{2 \pi} p^{n_{s}} d \vartheta_{0} \\
\times \exp \left[-i\left(\Delta_{s} \varsigma+\psi_{s}\right)\right] d \varphi \\
\frac{\partial p}{\partial \varsigma}+i\left(|p|^{2}-1\right) p=i \sum_{s}\left(p^{*}\right)^{n_{s}-1} \cdot f_{s} \exp \left[i\left(\Delta_{s} \varsigma+\psi_{s}\right)\right]
\end{gathered}
$$

Here, $p$ is the complex transverse momentum of the electron normalized to its initial absolute value, $\varsigma=\left(\beta_{\perp 0}^{2} \omega / 2 \beta_{\| 0} c\right) z$ is the dimensionless coordinate, $\beta_{\perp 0}=v_{\perp 0} / c$ and $\beta_{\| 0}=v_{\| 0} / c$ are normalized electron velocities, $n_{s}$ is the harmonic number, $\Delta_{s}=2\left(\omega-n_{s} \omega_{c}\right) /\left(\beta_{\perp 0}^{2} \omega\right)$ is the frequency mismatch, $s$ is the mode number, $\omega_{c} / 2 \pi=28 B / \gamma_{\text {rel }}$ is the electron cyclotron frequency in $\mathrm{GHz}, B$ is the magnetic field in $T, \gamma_{\text {rel }}=1+U / 511$ is the relativistic factor of electrons, $U$ is the accelerating voltage in $\mathrm{kV}, f_{s}(\varsigma, \tau)$ is the high-frequency field in the resonator, $\tau=\left(\beta_{\perp 0}^{4} / 8 \beta_{z 0}^{2}\right) \omega_{c} t$ is the dimensionless time, $\delta_{s}$ $=8 \beta_{\| 0}^{2} \beta_{\perp 0}^{-4}[\bar{\omega}-\omega(\varsigma)] \omega_{c}^{-1}$ describes variation of the critical frequency $\omega(\varsigma)$ along the resonator axis, $\bar{\omega}$ is the cutoff frequency of the straight section of the resonator, $\psi_{s}$ $=8 \beta_{\|}^{2} \beta_{\perp}^{-4}\left(\bar{\omega}_{s}-\omega_{c}\right) \omega_{c}^{-1} \tau+\left(n_{s} \mp m_{s}\right) \varphi$ is the phase of the mode, $\varphi$ is the azimuthal coordinate, and $I_{s}$ is the dimensionless current

$$
\begin{aligned}
I_{s}= & 3.76 \times 10^{-3} I_{0} \beta_{\| 0} \beta_{\perp 0}^{2\left(n_{s}-4\right)} \frac{J_{m_{s} \pm n_{s}}^{2}\left(\frac{2 \pi}{\lambda_{s}} R_{e l}\right)}{\gamma_{r e l}\left(\nu_{s}^{2}-m_{s}^{2}\right) J_{m}^{2}\left(\nu_{s}^{2}\right)} \\
& \cdot n_{s}^{3}\left(\frac{n_{s}^{n_{s}}}{2^{n_{s}} \cdot n_{s} !}\right)^{2} .
\end{aligned}
$$

Here, $I_{0}$ is current in amperes, $J$ is the Bessel function, $m$ is the azimuthal index of the mode, $\lambda$ is the wavelength, $R_{e l}$ is the electron beam radius, and $\nu$ is the zero of the derivative of the Bessel function. 
TABLE I. Frequencies and quality factors.

\begin{tabular}{lcc}
\hline \hline Mode & Frequency $(\mathrm{GHz})$ & $\mathrm{Q}$ \\
\hline 7,3 & 95.8 & 1123 \\
3,4 & 84.5 & 873 \\
6,4 & 107.9 & 1469 \\
\hline \hline
\end{tabular}

The system of Eqs. (1) and (2) has to be supplemented by the standard initial condition for the momentum $p(0)$ $=\exp \left(i \vartheta_{0}\right)$ with $0 \leq \vartheta_{0} \leq 2 \pi$, and by the boundary condition for the field at the entrance to the interaction space $f_{s}(0, \tau)=0$. At the exit from the interaction space $\left(\varsigma=\varsigma_{\text {out }}\right)$, the usual boundary condition is applied

$$
f_{s}\left(\varsigma_{\text {out }}, \tau\right)=\left.\frac{i}{k_{s}} \cdot \frac{\partial f_{s}(\varsigma, \tau)}{\partial \varsigma}\right|_{\varsigma=\varsigma_{\text {out }}} .
$$

Here, $k_{s}=2 c \beta_{\|} \beta_{\perp}^{-2} \omega_{c}^{-1}\left[\bar{\omega}^{2} / c^{2}-\nu_{s}^{2} / R_{c a v}^{2}\right]^{1 / 2}$ is the dimensionless axial wave number.

The efficiency is given by the expression

$$
\eta=\eta_{\perp} \cdot \frac{\alpha^{2}}{1+\alpha^{2}} \cdot \frac{\gamma_{\text {rel }}+1}{2 \gamma_{\text {rel }}},
$$

where

$$
\eta_{\perp}=1-\frac{1}{2 \pi} \int_{0}^{2 \pi}\left|p\left(\varsigma_{\text {out }}\right)\right|^{2} d \theta_{0} .
$$

The total power is defined as

$$
P_{\text {out }}=U \cdot I_{0} \cdot \eta
$$

The numerical implementation of this formalism is described in Ref. 2.

\section{NEW FORMALISM}

If the condition $t_{\text {transit }} \ll t_{\text {decay }}$ does not hold, or is marginally valid, Eq. (2) has to be modified (Ref. 5)

$$
\begin{aligned}
\frac{\alpha^{2}}{4} \cdot \frac{\partial p}{\partial \tau}+\frac{\partial p}{\partial \varsigma}+i\left(|p|^{2}-1\right) p= & i \sum_{s}\left(p^{*}\right)^{n_{s}-1} \\
& \cdot f_{s} \exp \left[i\left(\Delta_{s} \varsigma+\psi_{s}\right)\right] .
\end{aligned}
$$

Here, $\alpha=\beta_{\perp} / \beta_{\|}$is the electron pitch factor. The presence of the temporal derivative of $p$ means that the assumption about the frozen wave amplitude during the electron transit time is

TABLE II. Magnetic fields and frequency mismatches for the operating voltage.

\begin{tabular}{lccc}
\hline \hline $\mathrm{B}(\mathrm{T})$ & $\Delta_{7,3}$ & $\Delta_{3,4}$ & $\Delta_{6,4}$ \\
\hline 3.25 & 2.77 & 0.37 & 5.36 \\
3.60 & 0.79 & -1.37 & 3.13 \\
3.65 & 0.54 & -1.59 & 2.85 \\
3.70 & 0.29 & -1.81 & 2.57 \\
3.75 & 0.05 & -2.02 & 2.30 \\
3.80 & -0.18 & -2.22 & 2.04 \\
4.10 & -1.45 & -3.35 & 0.61 \\
\hline \hline
\end{tabular}

no longer valid. The existence of the first term in Eq. (8) greatly complicates numerical calculations [Ref. 1].

\section{NUMERICAL EXAMPLE}

To illustrate the difference between the results obtained by means of the two formalisms, we choose the gyrotron operating in the $T E_{7,3}^{-}$mode described in Ref. 6 . We consider two possible accompanying modes $T E_{3,4}^{-}$and $T E_{6,4}^{+}$. All three modes operate at the fundamental harmonic $n_{s}=1$.

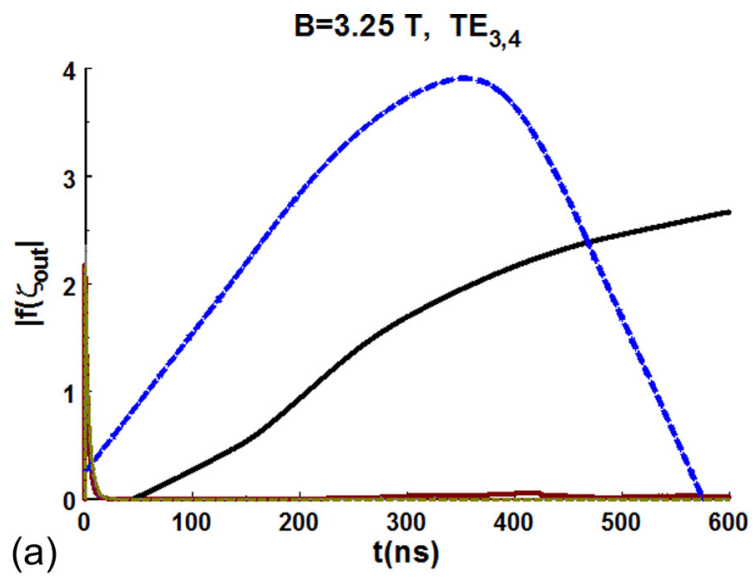

$\mathrm{B}=3.25 \mathrm{~T}, \mathrm{TE}_{3,4}$
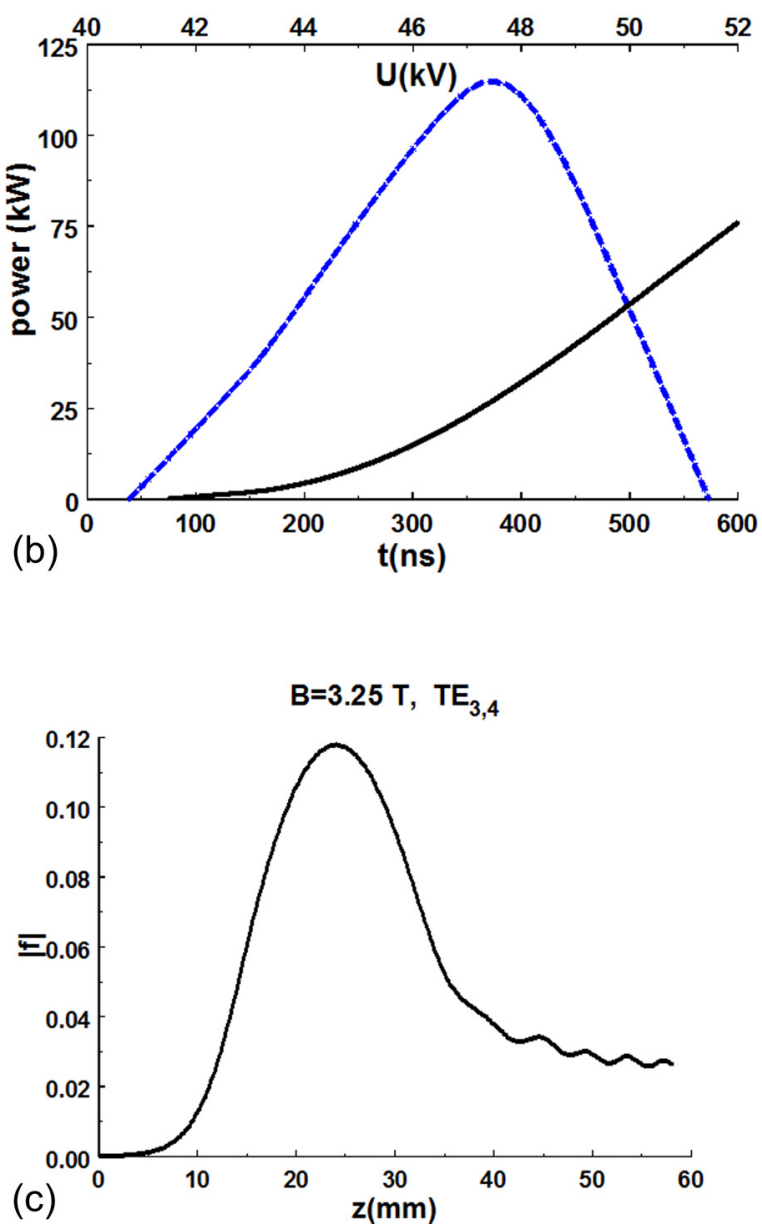

FIG. 1. (a) Field amplitude. (b) Output power. (c) Field profile. 

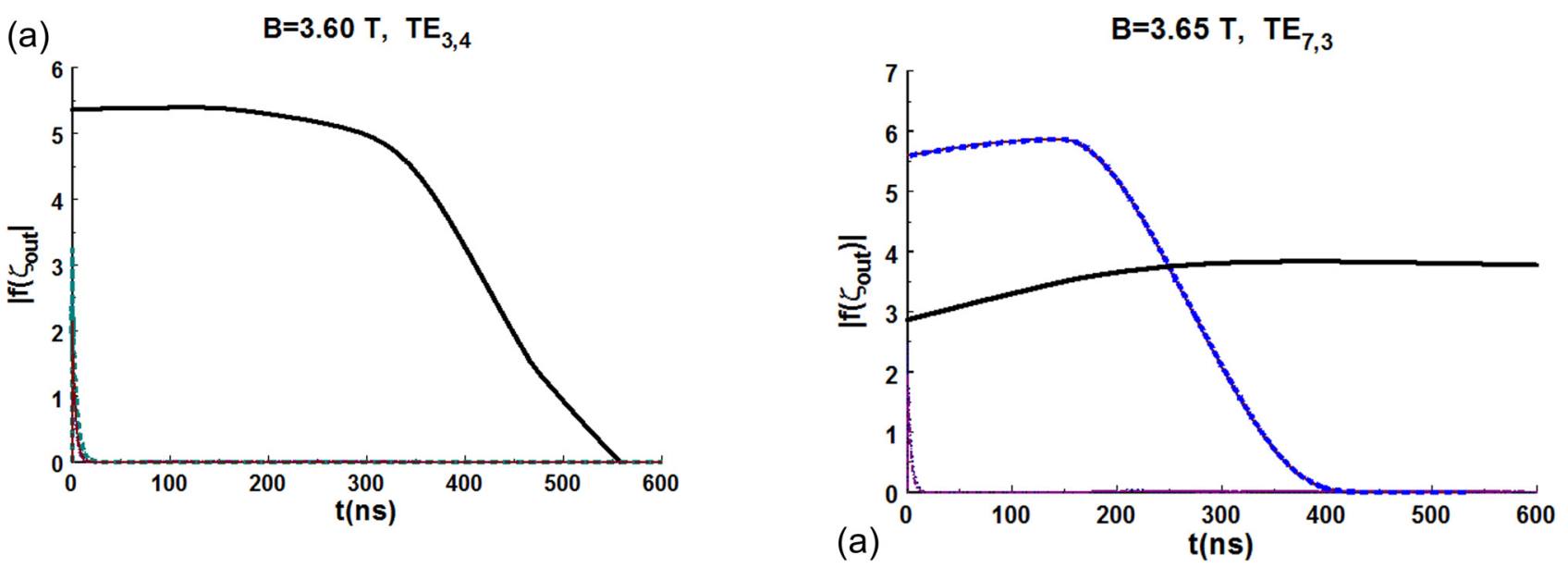

(b)

$\mathrm{B}=3.60 \mathrm{~T}, \mathrm{TE}_{3,4}$

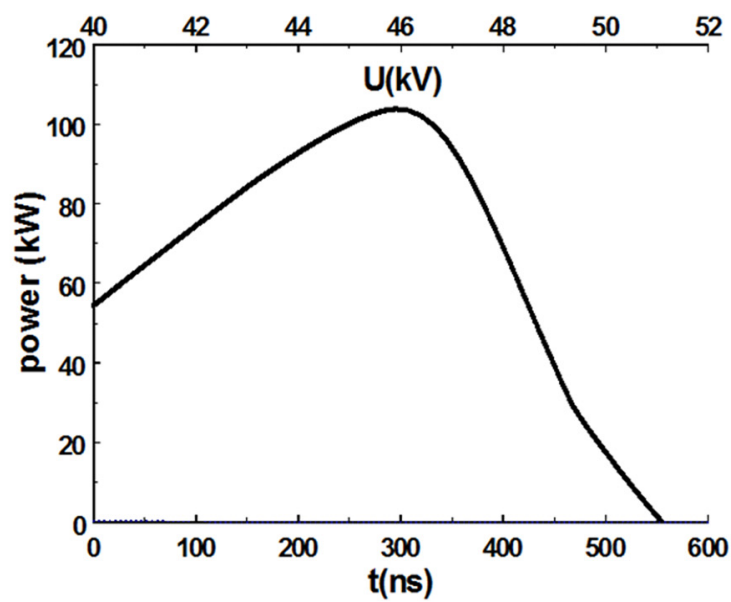

(c)

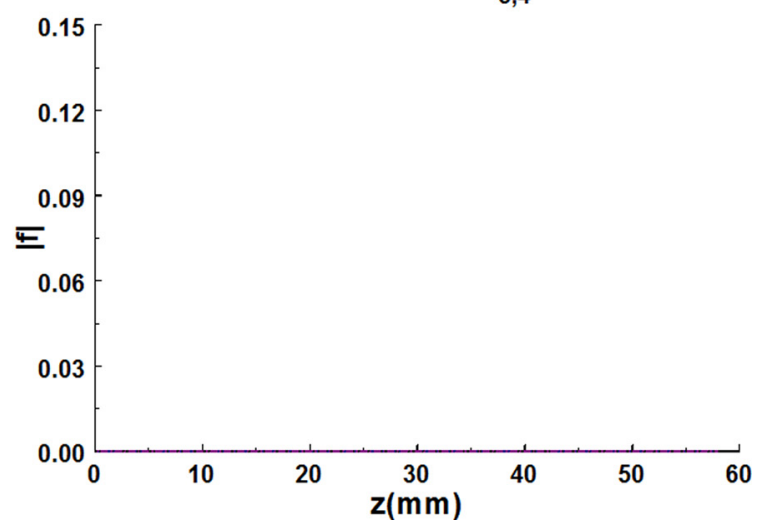

FIG. 2. (a) Field amplitude. (b) Output power. (c) Field profile.

The geometry of the cavity is: $\theta_{1}=2.3^{\circ}, \theta_{2}=0^{\circ}$, $\theta_{3}=3.0^{\circ}, L_{1}=15 \mathrm{~mm}, L_{2}=20 \mathrm{~mm}, L_{3}=23 \mathrm{~mm}$, and $R_{\text {cav }}=8.25 \mathrm{~mm}$. The frequencies and quality factors of the modes are summarized in Table I.

The operating parameters are: $U=52 \mathrm{kV}, I=7.4 \mathrm{~A}$, $\alpha=1.35$, and $R_{\text {beam }}=3.75 \mathrm{~mm}$.

We consider seven values of the magnetic field shown in Table II. Calculations start at $40 \mathrm{kV}$ and continue with the
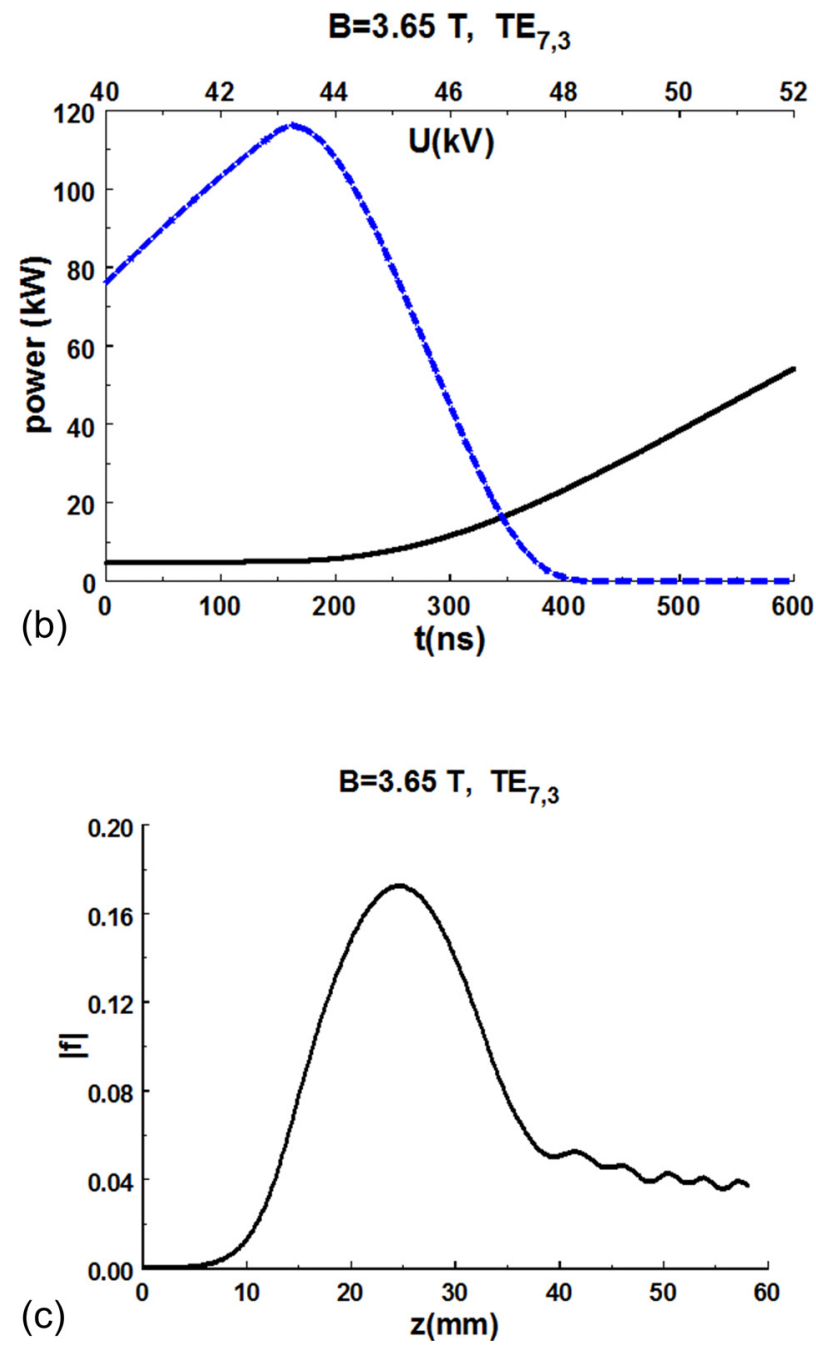

FIG. 3. (a) Field amplitude. (b) Output power. (c) Field profile.

step $0.5 \mathrm{kV}$ until the voltage $52 \mathrm{kV}$ is reached. Here at each voltage, calculations are carried out for $25 \mathrm{~ns}$. It was checked numerically that during this time stationary states were achieved for each voltage and for all modes. Thus, the total time of computation was equal to $2 \times 12 \times 25=600 \mathrm{~ns}$.

It should be emphasized that the edge magnetic fields $B$ $=3.25 \mathrm{~T}$ and $B=4.10 \mathrm{~T}$ were chosen only for illustrative 


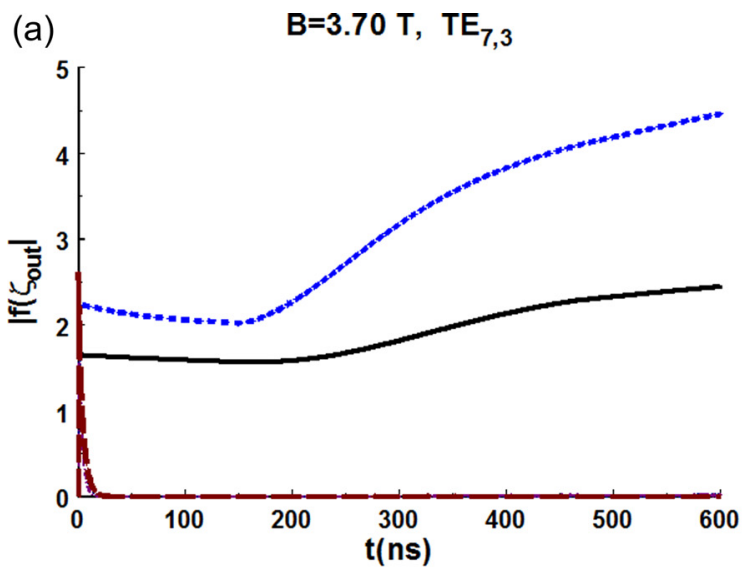

(b)

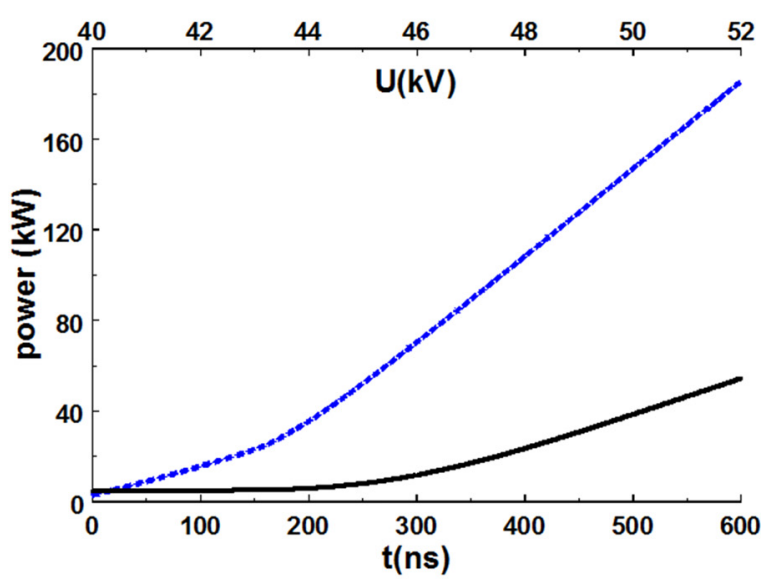

(c)

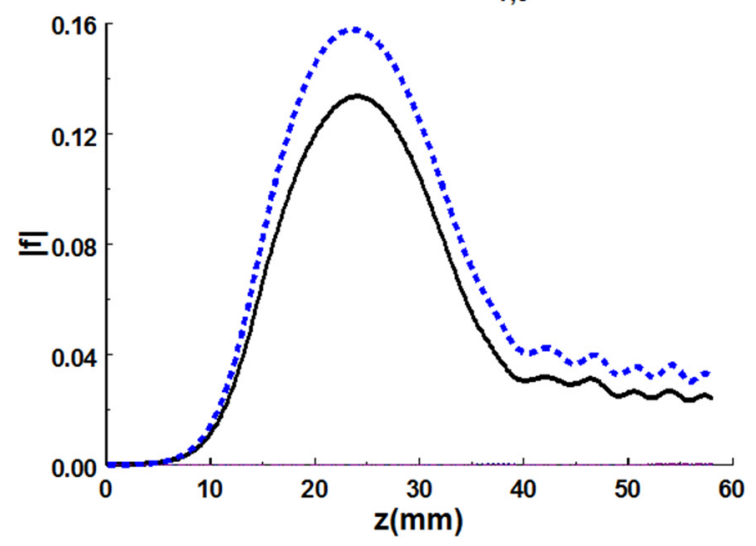

FIG. 4. (a) Field amplitude. (b) Output power. (c) Field profile.

purposes. It is obvious that at these magnetic fields the operating $T E_{7,3}$ mode cannot be excited, because its frequency mismatch is far beyond the "reasonable" values $0 \leq \Delta \leq 0.6$ (Ref. 7).

In the figures for each magnetic field, we show absolute values of the field amplitudes at the exit from the resonator $\left|f_{s}\left(\varsigma_{\text {out }}\right)\right|$. The total output power and the field profiles in the resonator correspond to the operating voltage $U=52 \mathrm{kV}$.

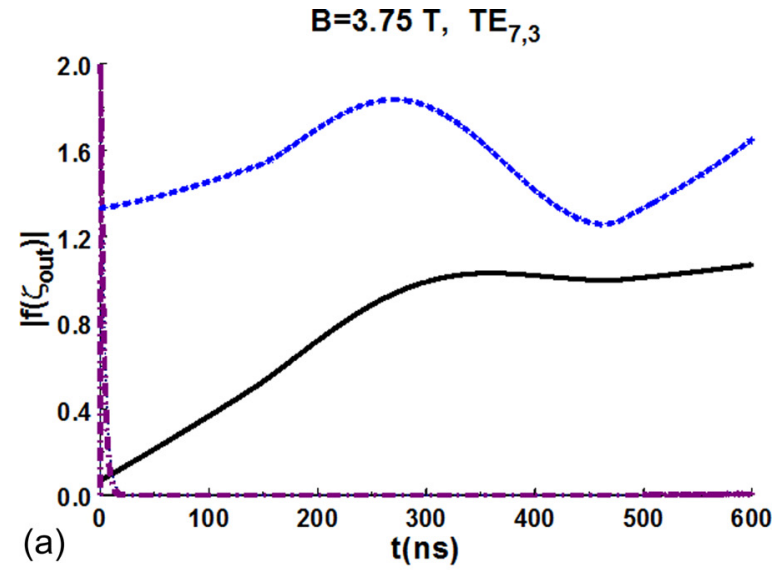

$\mathrm{B}=3.75 \mathrm{~T}, \mathrm{TE}_{7,3}$

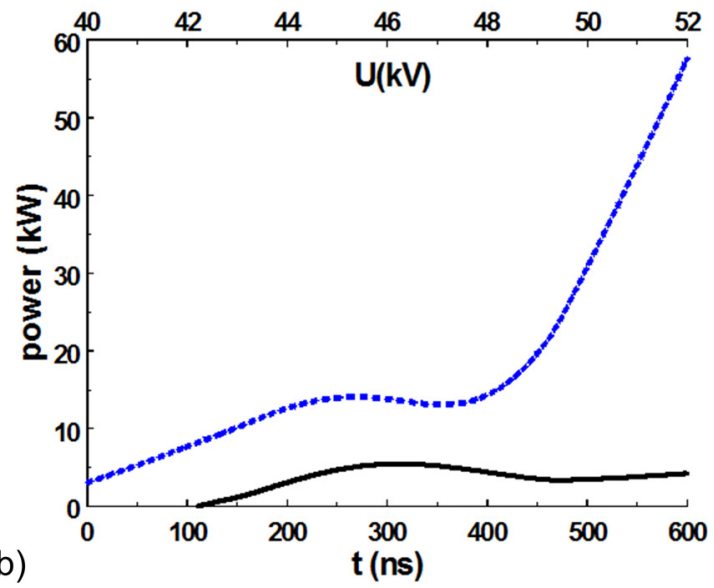

$B=3.75 T, T_{7,3}$

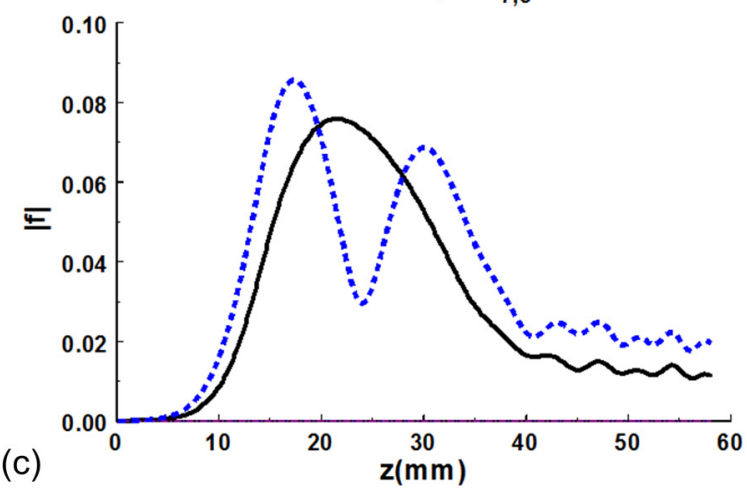

FIG. 5. (a) Field amplitude. (b) Output power. (c) Field profile.

The results obtained with the old formalism are displayed with solid black lines and those obtained by means of the new formalism with dashed blue lines.

\section{A. Magnetic field $B=3.25 \mathrm{~T}$}

As follows from Table II, at this magnetic field only oscillations in the mode $T E_{3,4}$ are expected (Fig. 1).

The old formalism predicts oscillations with a relatively high output power $>75 \mathrm{~kW}$. Note that beyond $600 \mathrm{~ns}$ $(U>52 \mathrm{kV})$ even a higher output power is expected. The 

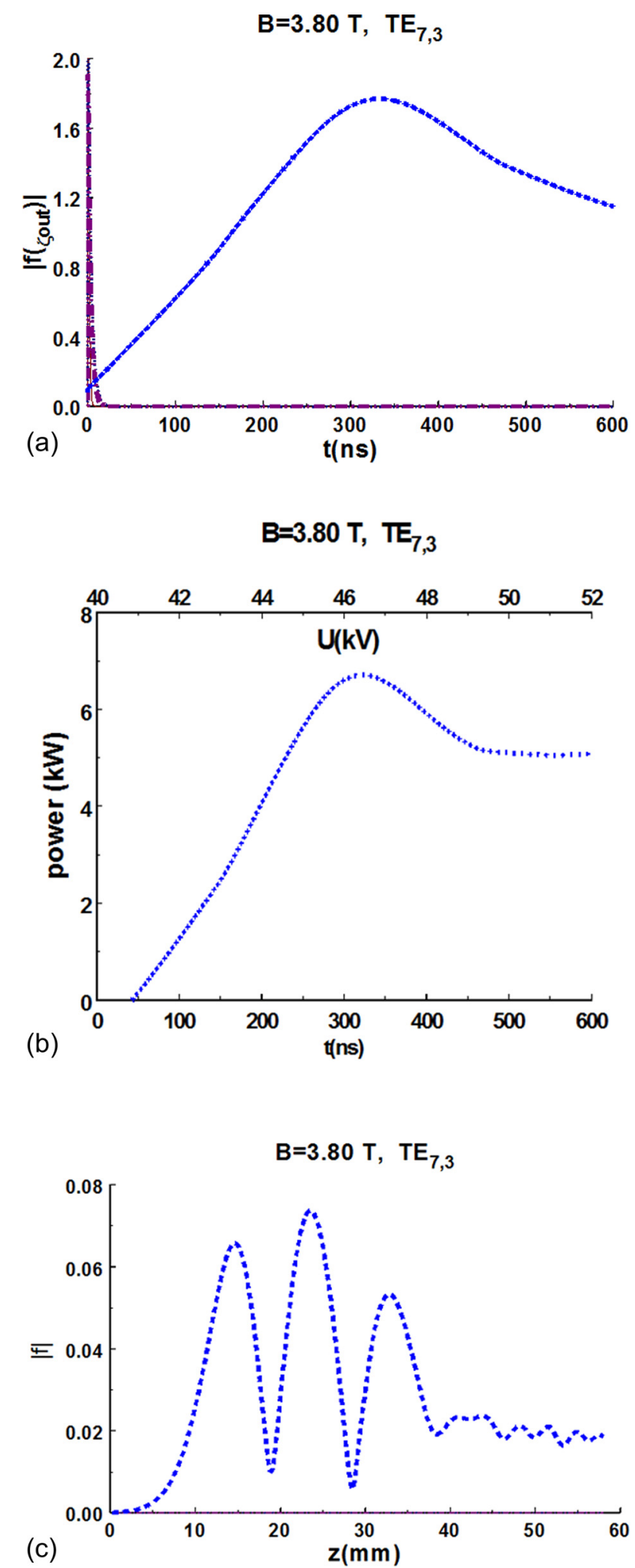

FIG. 6. (a) Field amplitude. (b) Output power. (c) Field profile.

new formalism predicts maximum power $\sim 115 \mathrm{~kW}$ at $370 \mathrm{~ns}$ $(47.5 \mathrm{kV})$. At higher voltages, the oscillations collapse.

\section{B. Magnetic field $B=3.60 \mathrm{~T}$}

At this magnetic field, no oscillations are expected at the operating voltage, because the frequency mismatch is not favorable for any mode.
It is interesting that the new formalism does not predict oscillations even at lower voltages. Figure 2(c) is "empty" because there are no oscillations.

\section{Magnetic field $B=3.65 \mathrm{~T}$}

At this magnetic field, only oscillations in the $T E_{7,3}$ mode are expected (Fig. 3).

The old formalism predicts low power oscillations at the nominal voltage, while the new formalism predicts high power oscillations at $43 \mathrm{kV}$, and no oscillations at the operating voltage.

(a)
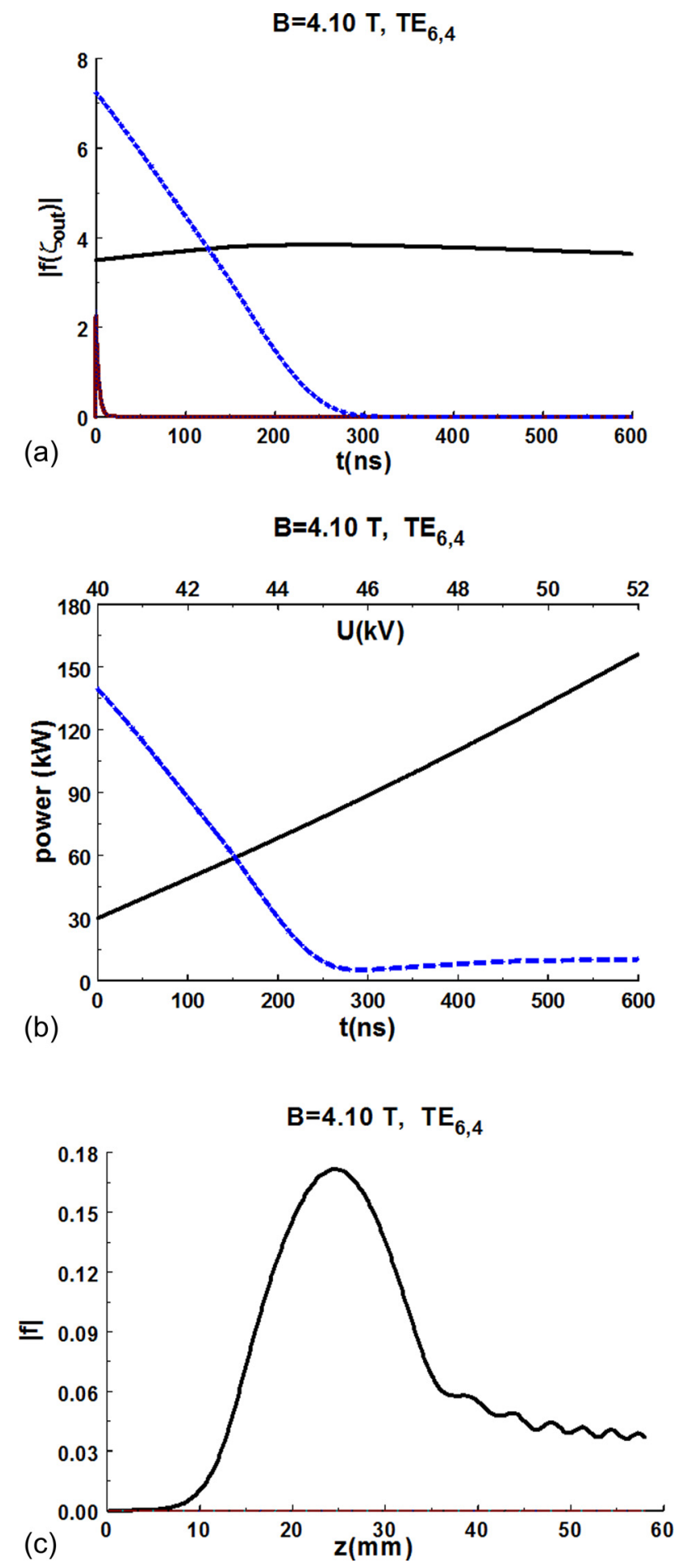

FIG. 7. (a) Field amplitude. (b) Output power. (c) Field profile. 


\section{Magnetic field $B=3.70 \mathrm{~T}$}

Also at this magnetic field, only oscillations in the $T E_{7,3}$ mode are expected (Fig. 4).

In this case, both the old and the new formalisms predict oscillations, albeit with different output powers. Note that the power increases for voltages higher than the operating voltage.

\section{E. Magnetic field $B=3.75 \mathrm{~T}$}

At this magnetic field, low power oscillations only in the $T E_{7,3}$ mode are expected (Fig. 5).

Here, again the two formalisms predict low power oscillations at the operating voltage. It is interesting that the new formalism yields the field profile with two peaks. This indicates that we are approaching the backward wave oscillation regime (BWO) $\Delta \leq 0$.

\section{F. Magnetic field $B=3.80 \mathrm{~T}$}

At this magnetic field, the frequency mismatch is negative. Very low power oscillations are expected (Fig. 6).

The old formalism predicts no oscillations. The three peaks in the field profile manifest that BWO is observed $(\Delta<0)$.

\section{G. Magnetic field $B=4.10 \mathrm{~T}$}

From Table II, it is obvious that at this magnetic field only oscillations in the $T E_{6,4}$ mode are possible (Fig. 7).

The old formalism predicts high power oscillations at the operating voltage, while the new formalism shows high power oscillations at lower voltages.

Note that in all figures showing the field amplitudes a suppression of competing modes is seen at $t \leq 15 \mathrm{~ns}$.

\section{CONCLUSIONS}

Behavior of oscillations predicted by the two formalisms in most cases is quite different. This indicates that the additional term in the equation of motion of electrons (8) plays a significant role.

It should be noted that the formalism described above is based on the system of equations (1) and (2) which was widely used in the former Soviet Union and nowadays still is used in Russia and in the USA. In Europe, powerful new gyrotron simulation codes based on the Particle-In-Cell (PIC) formalism have been developed (Refs. 8 and 9). In those codes, the approximation of time-frozen electromagnetic field during the electron transit across the cavity is relaxed. Essentially this should be equivalent to replacing Eq. (2) by Eq. (8). It would be interesting to check the results of the present study by means of the PIC simulations. This is beyond the scope of the present paper.

Finally, it should be noted that the relaxation of a timefrozen field during the electron transit through the cavity becomes more important in the following cases. (i) Competition between the fundamental and higher harmonics. The electron transit time through the resonator is one and the same for all harmonics, but the cavity decay time is shorter for the fundamental harmonic. (ii) Modes with higher axial indices. The condition $t_{\text {transit }} \ll t_{\text {decay }}$ holds better for lower axial indices. This might be important in the analysis of the frequency tunability based on the excitation of modes with different axial indices. (iii) Low voltage gyrotrons, when the electron transit time through the resonator is long.

In all these cases, the new formalism, or, alternatively, the PIC codes should be used.

\section{ACKNOWLEDGMENTS}

The work of H.K. was supported by the European Regional Development Funding of the Project No. 1.1.1.1/ 16/A/004.

${ }^{1}$ O. Dumbrajs and H. Kalis, Phys. Plasmas 22, 053113 (2015).

${ }^{2}$ M. I. Airila, O. Dumbrajs, A. Reinfelds, and U. Strautins, Phys. Plasmas 8 , 4608 (2001)

${ }^{3}$ O. Dumbrajs and G. S. Nusinovich, Phys. Plasmas 23, 083125 (2016)

${ }^{4}$ N. A. Zavolsky, G. S. Nusinovich, and A. B. Pavelyev, Girotrony (Gorky, 1989), p. 84 (in Russian).

${ }^{5}$ N. S. Ginzburg, A. S. Sergeev, and I. V. Zotova, Phys. Plasmas 22, 033101 (2015).

${ }^{6}$ N. Kumar, U. Singh, A. Kumar, and A. K. Sinha, Vacuum 99, 99 (2014).

${ }^{7}$ G. S. Nusinovich, Introduction to the Physics of Gyrotrons (The Johns Hopkins University Press, Baltimore and London, 2004), p. 74.

${ }^{8}$ F. Braunmueller, T. M. Tran, Q. Vuillemin, S. Alberti, J. Genoud, J.-P. Hogge, and M. Q. Tran, Phys. Plasmas 22, 063115 (2015).

${ }^{9}$ K. A. Avramidis, Z. C. Ioannidis, S. Kern, A. Samartsev, I. G. Tigelis, and J. Jelonnek, Phys. Plasmas 22, 053106 (2015). 